\title{
Novel Scoring System for Distinction Between Paroxysmal and Non-Paroxysmal Atrial Fibrillation
}

\author{
Jun Oikawa, MD; Shinichi Niwano, MD; Hidehira Fukaya, MD; Hironori Nakamura, MD; \\ Tazuru Igarashi, MD; Tamami Fujiishi, MD; Naruya Ishizue, MD; Tomoharu Yoshizawa, MD; \\ Akira Satoh, MD; Jun Kishihara, MD; Masami Murakami, MD; Junya Ako, MD
}

\begin{abstract}
Background: Distinction of paroxysmal atrial fibrillation (PAF) from non-PAF is important in clinical practice, but this is often difficult at the time of first documented AF. Given that fibrillation cycle length $(F C L)$ is longer in PAF than in non-PAF, the aim of this study was to compare various clinical parameters including FCL to establish a scoring system to distinguish PAF and non-PAF.
\end{abstract}

\begin{abstract}
Methods and Results: The subjects consisted of 382 consecutive patients with AF on digital ECG at the present institute between 2008 and 2011. They were divided into PAF and non-PAF groups according to the following clinical course. Propensity score matching yielded 88 matched patient pairs with similar mean age and gender between the 2 groups. FCL was evaluated using customized fibrillation wave analyzer with fast Fourier transform analysis. On multivariate analysis, higher HR, longer FCL, and smaller LAD were independent predictors of PAF. For the scoring, cut-offs for each parameter were determined according to highest sensitivity and specificity on the ROC curves, and 1 point assigned for each parameter. Using this scoring system, 2 points detected PAF with $64 \%$ sensitivity and $84 \%$ specificity.
\end{abstract}

Conclusions: We propose a scoring system including FCL to distinguish PAF from non-PAF. Further studies are needed to validate the results.

Key Words: Fast Fourier transform; Paroxysmal atrial fibrillation; Score system

A trial fibrillation (AF) is the most common tachyarrhythmia; ${ }^{1}$ it is defined as ventricular response regulated by a refractory atrioventricular node. AF is associated with a high risk of stroke, death, and heart failure. ${ }^{2}$ The prevalence of AF is related to aging, and approximately 2.2 million people in the USA have AF. In the Framingham study, the prevalence of AF was $2.3 \%$ in people aged $>40$ years, and $5.9 \%$ in those aged $>65$ years. ${ }^{3}$ Recent guidelines classified AF into 4 categories: first documented; paroxysmal; persistent; and permanent. ${ }^{4}$ The latter 3 can be identified only retrospectively according to the clinical course. Anticoagulation is indicated regardless of clinical course, ${ }^{5}$ but the primary treatment may be different. In the case of paroxysmal AF (PAF), rhythm control treatment, including prescription of anti-arrhythmic agents or simple pulmonary vein isolation, is more effective than in the other clinical types of AF. In contrast, in nonPAF including persistent $\mathrm{AF}$ and permanent $\mathrm{AF}$, antiarrhythmic agents are less effective and treatment is complicated by the necessity for catheter ablation. Rate control therapy can be simply chosen depending on AF patient clinical status. Therefore, distinction between PAF and non-PAF is important with regard to treatment deci- sion making.

Distinction between PAF and non-PAF, however, is difficult at the first documented AF episode. Palpitation is an important factor in the differentiation of clinical $\mathrm{AF}$ type, but there is a considerable number of patients with asymptomatic AF, therefore there is a limit to the determination of clinical type on symptoms. We have previously shown the clinical importance of atrial electrophysiological properties on analysis of fibrillation cycle length (FCL) ${ }^{6}$ This method enables non-invasive and repeated evaluation of the degree of refractory shortening or the complexity of the AF substrate, and provides indirect but important information on the degree of atrial remodeling. ${ }^{7}$ We have previously reported that PAF tended be associated with longer FCL than persistent AF, but such a distinction still carries considerable error when using FCL as the only predicting index. ${ }^{8}$ In the present study, we hypothesized that a combination of clinical parameters including FCL could distinguish PAF and non-PAF with acceptable sensitivity and specificity. The aim of this study was therefore to establish a scoring system for the distinction of PAF and non-PAF in patients with first documented AF.

Received October 24, 2016; revised manuscript received January 25, 2017; accepted January 27, 2017; released online February 28, 2017 Time for primary review: 21 days

Department of Cardiovascular Medicine, Kitasato University School of Medicine, Sagamihara, Japan

Mailing address: Shinichi Niwano, MD, Department of Cardiovascular Medicine, Kitasato University School of Medicine, 1-15-1 Kitasato, Minami-ku, Sagamihara 252-0374, Japan. E-mail: shniwano@med.kitasato-u.ac.jp

ISSN-1346-9843 All rights are reserved to the Japanese Circulation Society. For permissions, please e-mail: cj@j-circ.or.jp 


\section{Methods}

\section{Subjects}

Between 2008 and 2011, a total of 85,536 12-lead electrocardiogram (ECG) recordings were stored in the digital ECG profiling system of Kitasato University Hospital. From these recordings, AF episodes were observed in 1,590 patients. Exclusion criteria were as follows: (1) prescription of class I or class III anti-arrhythmics;,10 (2) preceding AF episode before the ECG recording; or (3) unknown later clinical course (Figure 1). The study was conducted with the approval of the Ethics Committee of Kitasato University Hospital and written informed consent was obtained before data analysis.

\section{ECG Analysis}

In patients with $\mathrm{AF}$ on surface 12-lead ECG, the recording V1 lead was used for analysis. By subtracting the QRS-T complex using a template-matching algorithm, pure fibrillation waves were extracted. ${ }^{11}$ In the next step, the total 8-s recording was separated into 8 epochs with $50 \%$ overlap. The spectral analysis of frequency was performed for each epoch using 1,024-point fast Fourier transformation (FFT) with band-pass filtering and application of a Hamming window. In the analyzed data, the power spectra were quantified by measuring the peak frequency signal with the maximum magnitude derived from the epoch. The peak frequency of the spectrum in the $3-12-\mathrm{Hz}$ range was converted to a cycle length as a reciprocal, defined as the FCL, and representative FCL was calculated as an average of 8 epochs. ${ }^{12}$ Figure 2 shows representative recordings of PAF and non-PAF.

\section{Statistical Analysis}

Statistical analysis was performed using JMP 10 (SAS Institute, Cary, NC, USA). Continuous variables are presented as mean \pm SD and were compared using Student's t-test or Mann-Whitney U-test. Dichotomous variables are presented as percentages and were compared using the chisquared test. Multivariate analysis was performed using

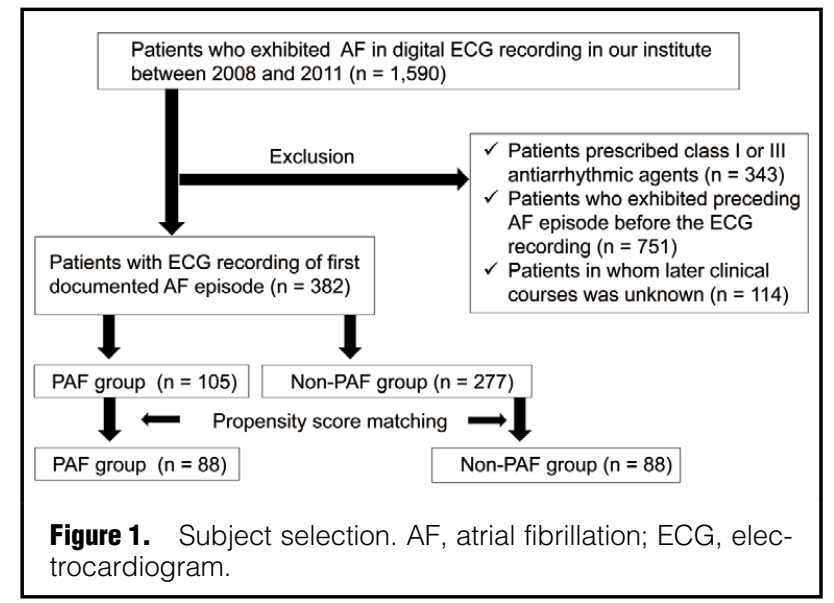

parameters that were significantly different on univariate analysis to determine independent factors predictive of PAF and non-PAF. Next, cut-offs for prediction of PAF were determined using receiver operating characteristic (ROC) analysis by optimizing the sensitivity and specificity for each parameter. Using these cut-offs, we then constructed a PAF score. $\mathrm{P}<0.05$ was considered to be statistically significant.

\section{Results}

\section{Subject Selection}

Three hundred and eighty-two patients were diagnosed with first documented AF episode. Mean age was $67 \pm 12$ years, 126 were female, and the mean FCL was $162.7 \pm 29.8 \mathrm{~ms}$. There was no apparent underlying disease in 88 of the AF patients. The 382 patients were then further divided into a PAF and non-PAF group according to clinical course. To control for further potential confounding and selection bias, a propensity score-matching technique was used to select the control population according to age and gen-

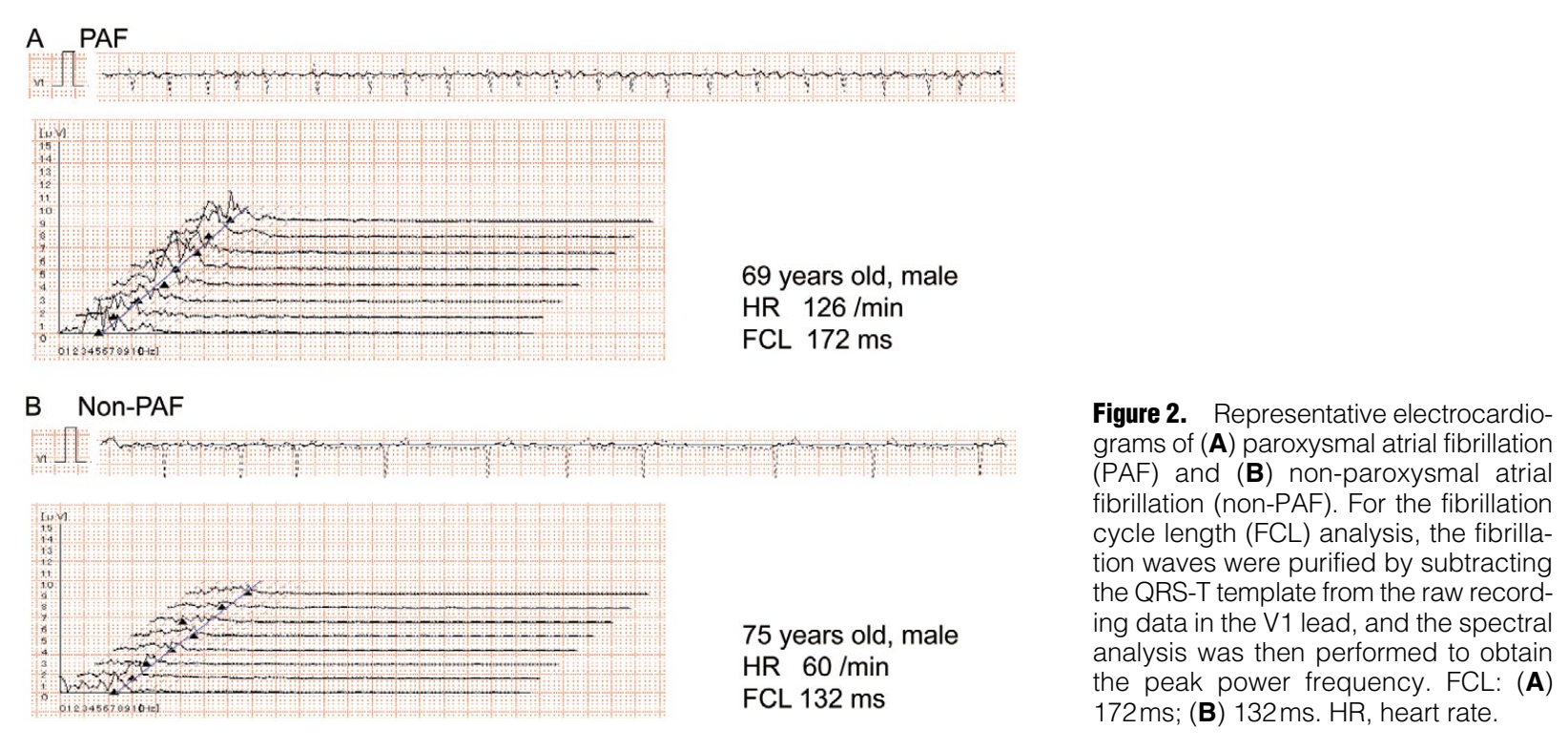




\begin{tabular}{|c|c|c|c|}
\hline & $\begin{array}{c}\text { PAF } \\
(n=88)\end{array}$ & $\begin{array}{c}\text { Non-PAF } \\
(n=88)\end{array}$ & $P$ value \\
\hline Age (years) & $70 \pm 10$ & $70 \pm 10$ & 1.00 \\
\hline Gender (M:F) & $59: 29$ & $59: 29$ & 1.00 \\
\hline Symptoms & $38(43)$ & $26(30)$ & 0.06 \\
\hline HR (beats/min) & $98 \pm 27$ & $84 \pm 22$ & $0.0003^{*}$ \\
\hline $\mathrm{SBP}(\mathrm{mmHg})$ & $126 \pm 27$ & $134 \pm 18$ & $0.04^{*}$ \\
\hline $\mathrm{DBP}(\mathrm{mmHg})$ & $77 \pm 15$ & $80 \pm 16$ & 0.18 \\
\hline $\mathrm{FCL}(\mathrm{ms})$ & $171.3 \pm 28.1$ & $150.7 \pm 21.2$ & $<0.0001^{*}$ \\
\hline \multicolumn{4}{|l|}{ Echocardiographic data } \\
\hline LAD (mm) & $42 \pm 8$ & $49 \pm 9$ & $<0.0001^{*}$ \\
\hline LVEF (\%) & $59.5 \pm 13.0$ & $59.5 \pm 13.2$ & 0.85 \\
\hline \multicolumn{4}{|l|}{ Laboratory data } \\
\hline $\mathrm{BNP}(\mathrm{pg} / \mathrm{mL})$ & $374.0 \pm 487.2$ & $249.1 \pm 246.8$ & 0.28 \\
\hline BUN (mg/dL) & $20.3 \pm 12.9$ & $20.3 \pm 13.1$ & 0.58 \\
\hline $\mathrm{Cr}(\mathrm{mg} / \mathrm{dL})$ & $1.17 \pm 1.35$ & $1.21 \pm 1.46$ & 0.17 \\
\hline LDL-C (mg/dL) & $107.3 \pm 38.9$ & $111.6 \pm 35.3$ & 0.47 \\
\hline $\mathrm{HbA1c}(\%)$ & $5.9 \pm 1.5$ & $6.0 \pm 1.1$ & 0.74 \\
\hline \multicolumn{4}{|l|}{ Underlying disease } \\
\hline $\mathrm{CHD}$ & $18(20)$ & $21(24)$ & 0.59 \\
\hline Valvular heart disease & $4(5)$ & $4(5)$ & 1.00 \\
\hline Hypertension & $52(59)$ & $59(67)$ & 0.42 \\
\hline Diabetes mellitus & $26(30)$ & $16(18)$ & 0.06 \\
\hline \multicolumn{4}{|l|}{ Incidence of prescription } \\
\hline ARB/ACEI & $32(36)$ & $28(32)$ & 0.45 \\
\hline$\beta$-blockers & $24(27)$ & $23(26)$ & 0.79 \\
\hline $\mathrm{CCB}$ & $24(27)$ & $25(28)$ & 0.94 \\
\hline Statins & $21(24)$ & $11(13)$ & $0.04^{*}$ \\
\hline
\end{tabular}

Data given as $\mathrm{n}(\%)$ or mean $\pm \mathrm{SD}$. ACEI, angiotensin-converting enzyme inhibitor; ARB, angiotensin receptor blocker; BNP, brain natriuretic peptide; BUN, blood urea nitrogen; $\mathrm{CCB}$, calcium-channel blockers; $\mathrm{CHD}$, congestive heart failure; DBP, diastolic blood pressure; FCL, fibrillation cycle length; Hb, hemoglobin; HR, heart rate; LAD, left atrial dimension; LDL-C, low-density lipoprotein cholesterol; LVEF, left ventricular ejection fraction; PAF, paroxysmal atrial fibrillation; SBP, systolic blood pressure.

\begin{tabular}{|lccc|}
\hline Table 2. Multivariate Indicators of PAF & & & \\
& OR & $95 \% \mathbf{C l}$ & P value \\
Heart rate & 1.02 & $1.004-1.039$ & $0.014^{*}$ \\
SBP & 0.99 & $0.973-1.007$ & 0.243 \\
FCL & 1.048 & $1.028-1.072$ & $<0.0001^{*}$ \\
LAD & 0.917 & $0.868-0.963$ & $0.0004^{*}$ \\
Statins & 0.629 & $0.220-1.700$ & 0.3543 \\
\hline
\end{tabular}

${ }^{*} \mathrm{P}<0.05 . \mathrm{Cl}$, confidence interval; OR, odds ratio. Other abbreviations as in Table 1.

der. ${ }^{13}$ Finally, 88 patients with PAF episodes and 88 ageand gender-matched non-PAF (i.e., control) patients were selected, and defined as the PAF and non-PAF groups, respectively (Figure 1). Clinical background, echocardiogram data, laboratory data, and FCL were compared between the 2 groups.

\section{PAF vs. Non-PAF}

After applying the propensity score matching, there were 88 matched pairs of PAF and non-PAF subjects. Mean age and gender were not different between the 2 groups (Table 1). On comparison of the 2 groups, heart rate $(\mathrm{HR} ; \mathrm{P}=0.0003)$, systolic blood pressure ( $\mathrm{P}=0.04)$, FCL $(\mathrm{P}<0.0001)$, and left atrial dimension $(\mathrm{LAD})$ on echocardiography $(\mathrm{P}<0.0001)$ were significantly different. Underlying disease and pre- scribed medicine are also listed in Table 1. The incidence of statins prescription $(24 \%$ vs. $13 \%, \mathrm{P}=0.04)$ was higher in the PAF group than in the non-PAF group.

\section{Multivariate Analysis}

Table 2 summarizes the results of the multivariate analysis using the prevalence of prescribed medicines and clinical parameters that were significantly different on univariate analysis to identify independent factors for PAF and nonPAF. On multivariate analysis, higher HR, longer FCL, and smaller LAD were identified as independent factors for PAF.

\section{Detection of PAF}

On multivariate analysis, HR, FCL, and LAD were identi- 
A

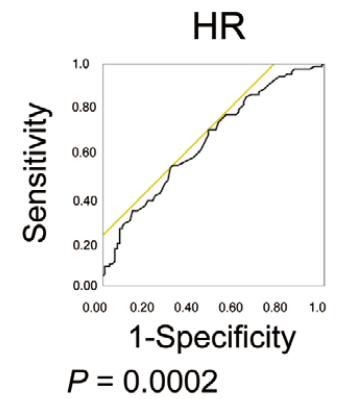

AUC 0.65

Sensitivity: $70 \%$ Specificity: $53 \%$

Cut off: $>83 / \mathrm{min}$
B

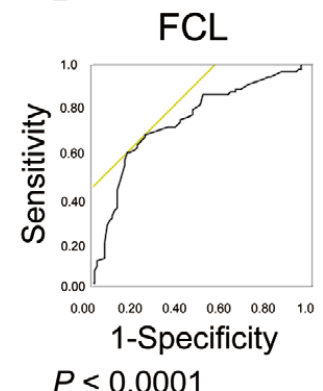

AUC 0.75

Sensitivity: $60 \%$ Specificity: $84 \%$

Cut off: $>165 \mathrm{~ms}$

C

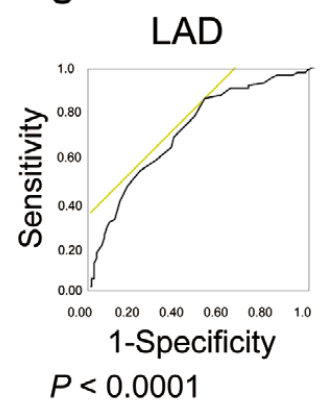

AUC 0.73

Sensitivity: $49 \%$ Specificity: $86 \%$

Cut off: $<41 \mathrm{~mm}$
Figure 3. Receiver operating characteristic (ROC) curves for the detection of first documented atrial fibrillation (AF) using significant multivariate predictors: (A) heart rate (HR); (B) fibrillation cycle length (FCL); and (C) left atrial dimension (LAD). Arrows, most powerful cut-off points for detection of paroxysmal AF.

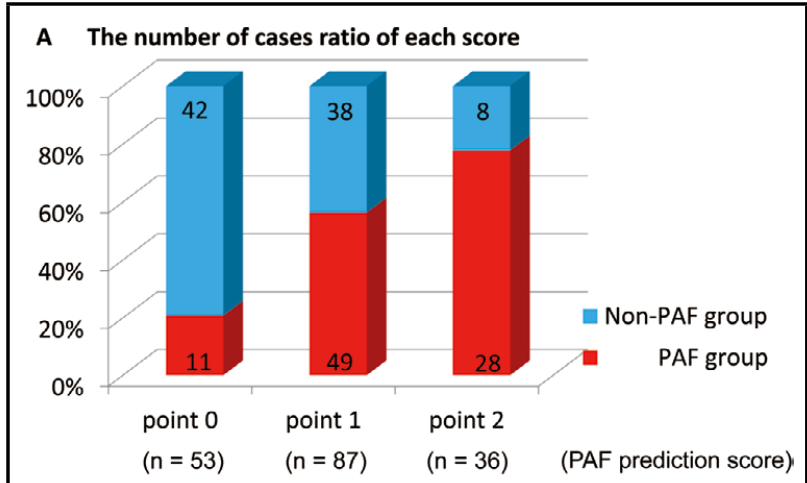

B Sensitivity and specificity of PAF prediction score

(\%)

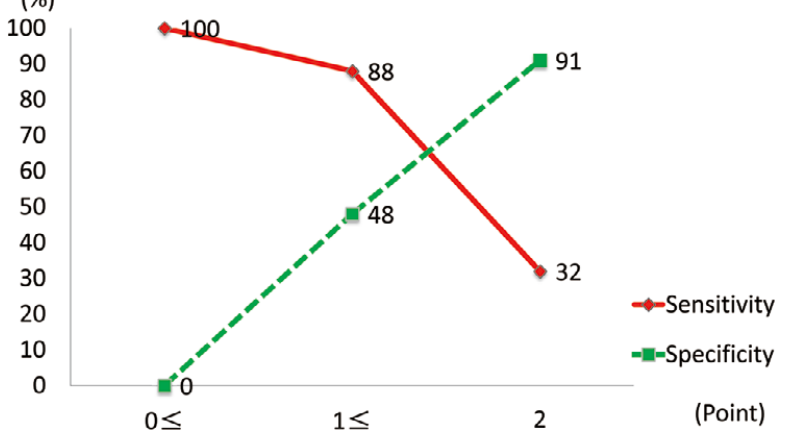

Figure 4. Two-parameter version of the paroxysmal atrial fibrillation (PAF) prediction score. (A) No. patients and proportion of PAF to non-PAF according to PAF score. (B) Sensitivity and specificity of PAF prediction score for the detection of PAF. For example, when 1 point was set as the threshold for the detection of PAF, 87 (1 point)+36 (2 points) patients were predicted to have PAF, 49 (1 point)+28 (2 points) PAF patients were correctly diagnosed, but 11 (0 points) patients were misdiagnosed, then sensitivity was calculated as $88 \%$ (78/88). In contrast, 53 (0 points) patients were predicted not to have PAF, 42 (0 points) patients were correctly diagnosed, but 38 ( 1 point $)+8$ ( 2 points) were misdiagnosed, then specificity was calculated as $48 \%$ (42/88).
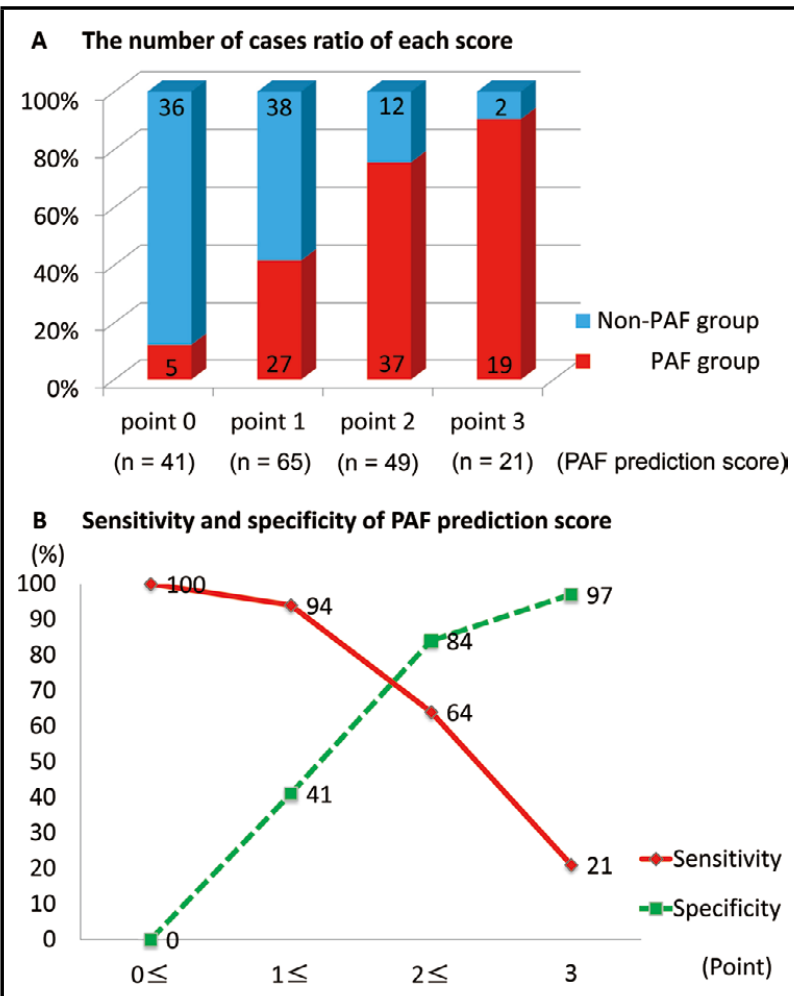

Figure 5. Three-parameter version of the paroxysmal atrial fibrillation (PAF) prediction score. (A) No. patients and proportion of PAF to non-PAF according to PAF score. (B) Sensitivity and specificity of PAF prediction score for the detection of PAF. Calculation method for sensitivity and specificity is given in Figure 4.

fied as independent factors. Cut-offs for prediction of PAF were determined on ROC curve analysis by optimizing sensitivity and specificity for each parameter (Figure 3). Using these 3 cut-offs for HR, FCL, and LAD, we constructed the PAF score. One point was scored when each criterion was met, with 3 points being the maximum. Initially, however, the PAF score was first constructed using 
HR and LAD without FCL, given that use of FFT to calculate FCL was not widespread at that time. Figure 4 shows the prediction of PAF using the 2-parameter PAF score. The sensitivity and specificity for detection of PAF at each score level was calculated by setting that point as the cut-off for detection of PAF. For example, when the cut-off was set at a score of 1 point, the patients with 1 and 2 points were predicted to have PAF, whereas the patients with 0 points were predicted to have non-PAF, with a sensitivity of $88 \%$ and a specificity of $48 \%$ for PAF detection. In contrast, Figure 5 shows the prediction of PAF using the 3-parameter PAF score including FCL. Comparing Figure 4 (2 parameters) and Figure 5 (3 parameters), the 3-parameter version had lower sensitivity but higher specificity than the 2-parameter version, thus the 3-parameter version was judged to be better than the 2-parameter version.

\section{Discussion}

In the present study, FCL, higher HR, longer FCL, and smaller LAD were identified as independent factors for distinguishing PAF in a simple scoring system.

\section{Clinical Factors for Distinction of PAF}

On multivariate analysis, higher HR, longer FCL and smaller LAD were independent predictors for PAF in first documented AF. Because all parameters can be obtained on routine ECG or echocardiography, these are feasible indices for distinction of PAF.

First, frequent atrial activation during AF is conducted to the ventricle through the atrioventricular node, resulting in a rapid irregular rhythm. In the present study, PAF patients had a higher heart rate than non-PAF patients. The mechanism underlying this difference is unclear, but it might be due to a change in the atrioventricular node and/ or influence of autonomic tone. ${ }^{14}$ Several studies have demonstrated the association between higher heart rate and high frequent stimulation of autonomic nerves by the atrioventricular node. ${ }^{15-17}$ This process may cause downregulation of ion channels located in the atrioventricular node. Moreover, sympathetic tone can also be activated by AF, but such an effect would be attenuated in the case of frequent AF episodes due to habituation. ${ }^{15}$

Second, permanent and long-lasting AF will result in an increase in LA pressure and wall stress. Atrial hypertrophy, fibrosis, and mal-expression of gap junction are also caused by the structural remodeling in AF. ${ }^{16}$ Because PAF patients tend to have shorter clinical history than non-PAF patients, they may have smaller LA.

Finally, as previously discussed in the literature, FCL calculated from surface ECG is an indirect parameter reflecting not only the refractoriness but also the complexity of re-entrant circuits on both atria in AF.12,17,18 Relatively rapid shortening of FCL occurs at the very beginning of the AF episode as a result of electrical remodeling, hence PAF patients may have longer FCL than non-PAF patients, who in turn tend to have a longer history of AF than PAF patients. ${ }^{18}$

In contrast, the parameters reflecting general condition or other organ function were not identified as indicators of PAF and non-PAF distinction. This may be because the difference between PAF and non-PAF might be reflected in the electrical, structural and neurohumoral factors rather than in other factors reflecting long-term morbidity of the underlying disease, at least in the case of first documented AF.

\section{PAF Score}

Herein we have proposed a PAF score using 3 parameters identified on multivariate analysis to distinguish between PAF and non-PAF. We calculated the cut-offs of these 3 factors on ROC curve analysis to achieve the highest sensitivity and specificity. This scoring system achieved relatively high sensitivity and specificity for the distinction of $\mathrm{PAF}$ and non-PAF.

Several clinical trials including AFFIRM and AF-CHF have demonstrated no clinical benefit of rhythm control using anti-arrhythmic agents in AF patients, ${ }^{\mathbf{1 9}, 20}$ while J-RHYTHM noted a limited benefit of rhythm control therapy in quality of life, at least in PAF patients. ${ }^{21}$ In the AFFIRM subanalysis, however, $35 \%$ of the rate control group were in sinus rhythm. Although the use of rhythm control drugs was associated with increased mortality of $49 \%$, the presence of sinus rhythm was associated with decreased mortality by $53 \%{ }^{22}$ This indicates that the maintenance of sinus rhythm may be beneficial at least in AF patients in whom the maintenance of sinus rhythm is feasible. The present PAF score facilitates identification of PAF patients, who may benefit from rhythm control therapy, including pharmacological and catheter ablation.

More recently, Tsadok et al reported a comparison of stroke events between the rhythm and rate control groups in a propensity score-matched population. ${ }^{23}$ They found that the stroke risk was significantly lower in the rhythm control group than in the rate control group, and such a tendency was more prominent in patients with $\mathrm{CHADS}_{2}$ (congestive heart failure, hypertension, age $\geq 75$ years, diabetes mellitus, and previous stroke) $>1$. With regard to stroke risk, distinction of AF patients in whom rhythm control is more effective, is an important issue.

\section{Study Limitations}

This study has several limitations. First, the identification of first documented AF may include errors related to the underestimation of preceding AF episodes. Second, this study was retrospective; therefore, the use of anti-arrhythmic and/or rate control agents was left to physician discretion. Finally, the number of patients was limited, sampling bias could not be eliminated, and differences in the population may have influenced patient selection. These limitations should be addressed in future studies with a large number of patients.

\section{Conclusions}

A scoring system using HR, FCL, and LAD distinguished PAF and non-PAF patients with considerable sensitivity and specificity. This score may be useful in clinical practice to determine the initial therapy for first documented AF.

\section{Acknowledgments}

None.

\section{Disclosures}

The authors declare no conflict of interest.

\section{References}

1. Nattel S, Opie LH. Controversies in atrial fibrillation. Lancet 2006; 367: 262-272. 
2. Suzuki S, Yamashita T, Ohtsuka T, Sagara K, Uejima T, Oikawa $\mathrm{Y}$, et al. Prevalence and prognosis of patients with atrial fibrillation in Japan: A prospective cohort of Shinken Database. Circ J 2008; 72: 914-920.

3. Feinberg WM, Blackshear JL, Laupacis A, Kronmal R, Hart RG. Prevalence, age distribution, and gender of patients with atrial fibrillation. Arch Intern Med 1995; 155: 469-473.

4. January CT, Wann LS, Alpert JS, Calkins H, Cigarroa JE, Cleveland JC Jr, et al. 2014 AHA/ACC/HRS guideline for the management of patients with atrial fibrillation: A report of the American College of Cardiology/American Heart Association Task Force on practice guidelines and the Heart Rhythm Society. Circulation 2014; 130: 2071-2104.

5. Gage BF, Waterman AD, Shannon W, Boechler M, Rich MW, Radford MJ. Validation of clinical classification schemes for predicting stroke: Results from the National Registry of Atrial Fibrillation. JAMA 2001; 285: 2864-2870.

6. Husser D, Stridh M, Sornmol L, Olsson SB, Bollmann A. Frequency analysis of atrial fibrillation from the surface electrocardiogram. Indian Pacing Electrophysiol J 2004; 4: 122-136.

7. Sasaki T, Niwano S, Sasaki S, Imaki R, Yuge M, Hirasawa S, et al Long-term follow-up of changes in fibrillation waves in patients with persistent atrial fibrillation: Spectral analysis of surface ECG. Circ J 2006; 70: 169-173.

8. Aoyama Y, Niwano S, Niwano H, Satoh A, Kishihara J, Ishikawa $\mathrm{S}$, et al. Repetitive evaluation of fibrillation cycle length predicts the efficacy of bepridil for interruption of long-lasting persistent atrial fibrillation. Int Heart J 2011; 52: 353-358.

9. Fujiki A, Nakagawa H, Sakabe M, Sakurai K, Nishida K, Mizumaki K, et al. Spectral characteristics of human atrial fibrillation waves of the right atrial free wall with respect to the duration of atrial fibrillation and effect of class I antiarrhythmic drugs. Jpn Circ J 2001; 65: 1047-1051.

10. Niwano S, Sasaki T, Kurokawa S, Kiryu M, Fukaya H, Hatakeyama Y, et al. Predicting the efficacy of antiarrhythmic agents for interrupting persistent atrial fibrillation according to spectral analysis of the fibrillation waves on the surface ECG. Circ J 2009; 73: 1210-1218.

11. Fujiki A, Sakabe M, Nishida K, Mizumaki K, Inoue H. Role of fibrillation cycle length in spontaneous and drug-induced termination of human atrial fibrillation. Circ J 2003; 67: 391 - 395 .

12. Oikawa J, Niwano S, Niwano H, Ishizue N, Yoshizawa T, Satoh A, et al. Prophylactic statin administration may prevent shortening of the fibrillation cycle length in patients with new-onset atrial fibrillation. Int Heart $J$ 2013; 54: 371-376.

13. Newgard CD, Hedges JR, Arthur M, Mullins RJ. Advanced statistics: The propensity score: A method for estimating treatment effect in observational research. Acad Emerg Med 2004; 11: $953-961$.

14. Toivonen L, Kadish A, Kou W, Morady F. Determinants of the ventricular rate during atrial fibrillation. J Am Coll Cardiol 1990; 16: $1194-1200$

15. Corino VD, Sandberg F, Mainardi LT, Sornmo L. An atrioventricular node model for analysis of the ventricular response during atrial fibrillation. IEEE Trans Biomed Eng 2011; 58: 3386-3395.

16. Nattel S, Danshi Li. Ionic remodeling in the heart: Pathophysiological significance and new therapeutic opportunities for atrial fibrillation. Circ Res 2000; 87: 440-447.

17. Neuberger HR, Schotten U, Blaauw Y, Vollmann D, Eijsbouts $\mathrm{S}$, van Hunnik A, et al. Chronic atrial dilation, electrical remodeling, and atrial fibrillation in the goat. J Am Coll Cardiol 2006; 47: $644-653$.

18. Watanabe I, Okumura Y, Nagashima K, Ohkubo K, Ashino S, Kofune M, et al. Electrical remodeling in fibrillating canine atrium: Action potential alternans during rapid atrial pacing and late phase 3 early afterdepolarization after cessation of rapid atrial pacing. Int Heart $J$ 2010; 51: 354-358.

19. Wyse DG, Waldo AL, DiMarco JP, Domanski MJ, Rosenberg Y, Schron EB, et al. A comparison of rate control and rhythm control in patients with atrial fibrillation. $N$ Engl J Med 2002; 347: $1825-1833$.

20. Roy D, Talajic M, Nattel S, Wyse DG, Dorian P, Lee KL, et al Rhythm control versus rate control for atrial fibrillation and heart failure. $N$ Engl J Med 2008; 358: 2667-2677.

21. Ogawa S, Yamashita T, Yamazaki T, Aizawa Y, Atarashi H, Inoue $\mathrm{H}$, et al. Optimal treatment strategy for patients with paroxysmal atrial fibrillation: J-RHYTHM Study. Circ J 2009; 73: $242-248$.

22. Corley SD, Epstein AE, DiMarco JP, Domanski MJ, Geller N, Greene HL, et al. Relationships between sinus rhythm, treatment, and survival in the Atrial Fibrillation Follow-Up Investigation of Rhythm Management (AFFIRM) Study. Circulation 2004; 109: $1509-1513$.

23. Tsadok MA, Jackevicius CA, Essebag V, Eisenberg MJ, Rahme $\mathrm{E}$, Humphries KH, et al. Rhythm versus rate control therapy and subsequent stroke or transient ischemic attack in patients with atrial fibrillation. Circulation 2012; 126: 2680-2687. 